\title{
A GENERALIZED CONVERSE MEASURABILITY THEOREM
}

\author{
K. S. CHANG AND K. S. RYU
}

(Communicated by R. Daniel Mauldin)

\begin{abstract}
It has long been known that measurability questions in Wiener space and Yeh-Wiener space are often rather delicate. Some converse measurability theorems in Wiener and Yeh-Wiener spaces were proved by Köehler, Skoug, and the first author.

In this paper, we establish a generalized converse measurability theorem by which the above measurability theorems are proved as corollaries.
\end{abstract}

1. Introduction. Let $C_{1}[a, b]$ denote the Wiener space of functions of one variable i.e., $C_{1}[a, b]=\{x(\cdot) \mid x(a)=0$ and $x(s)$ is continuous on $[a, b]\}$. Let $R=$ $\{(s, t) \mid a \leq s \leq b, \alpha \leq t \leq \beta\}$ and let $C_{2}[R]$ be the Yeh-Wiener space (or 2-parameter Wiener space), i.e., $C_{2}[R]=\{x(\cdot, \cdot) \mid x(a, t)=x(s, \alpha)=0, x(s, t)$ is continuous on $R\}$. Let $\nu$ be Wiener measure on $C_{1}[a, b]$ and let $\lambda$ be Yeh-Wiener measure on $C_{2}[R]$. For a discussion of Yeh-Wiener measure see [1, 5, and 6]. Note that Wiener space and Yeh-Wiener space are separable Banach spaces with respect to the supremum norm.

Let $a=t_{0}<t_{1}<\cdots<t_{n}=b$ be a subdivision of $[a, b]$. Let $E$ be any subset of Euclidean space $\mathbf{R}^{n}$ and define $J: C_{1}[a, b] \rightarrow \mathbf{R}^{n}$ by

$$
J(x)=\left(x\left(t_{1}\right), x\left(t_{2}\right), \ldots, x\left(t_{n}\right)\right) .
$$

$J$ is continuous on $C_{1}[a, b]$ with respect to the uniform topology. By the definition of Wiener measure, if $E$ is Lebesgue measurable then $J^{-1}(E)$ is Wiener measurable and

$$
\nu\left(J^{-1} E\right)=\int_{E} g(\vec{\xi}) d \gamma(\vec{\xi})
$$

where $\boldsymbol{\gamma}$ is Lebesgue measure on $\mathbf{R}^{n}$,

$$
\begin{aligned}
g(\vec{\xi})= & {\left[(2 \pi)^{n}\left(t_{1}-a\right)\left(t_{2}-t_{1}\right) \cdots\left(t_{n}-t_{n-1}\right)\right]^{-1 / 2} } \\
& \times \exp \left\{-\frac{1}{2} \sum_{i=1}^{n}\left(\xi_{i}-\xi_{i-1}\right)^{2} /\left(t_{i}-t_{i-1}\right)\right\}>0,
\end{aligned}
$$

and $\vec{\xi}=\left(\xi_{1}, \xi_{2}, \ldots, \xi_{n}\right) \in \mathbf{R}^{n}$.

In an unpublished result Köehler established the converse, i.e., if $J^{-1}(E)$ is Wiener measurable then $E$ is Lebesgue measurable. In [5], Skoug extended Köeh-

Received by the editors June $11,1987$.

1980 Mathematics Subject Classification (1985 Revision). Primary 28C20.

Key words and phrases. Wiener measure, Yeh-Wiener measure, tight measure, $n$-parallel lines theorem, converse measurability.

Research partially supported by Korea Science and Engineering Foundation, and Korean Ministry of Education. 
ler's result to Yeh-Wiener space and established the converse of the one line theorem in [1]. In [2], the first author extended Skoug's result to the $n$-parallel lines theorem in [1]. Later the proof of the converse of the $n$-parallel lines theorem was simplified [3].

In this paper, we establish a generalized converse measurability theorem which has the above converse measurability theorems as corollaries. The techniques used in this paper are quite different from the techniques used in [2, 3, and 5].

2. A generalized converse measurability theorem. A probability measure $P$ on a $\sigma$-algebra $\mathscr{S}$ containing the Borel sets in a topological space is called tight if for any $\varepsilon>0$ and for any $E$ in $\mathscr{S}$ there exists a compact set $K \subset E$ such that $P(F \backslash K)<\varepsilon$. It is known that any probability measure on the Borel class of a complete separable metric space is tight [4].

Let $P$ be a tight measure on $\mathscr{B}(S)$ and let $m$ be a measure on $\mathscr{B}(T)$ where $\mathscr{B}(S)$ and $\mathscr{B}(T)$ are the Borel classes of topological spaces $S$ and $T$, respectively. Let $(S, \overline{\mathscr{B}}(S), \bar{P})$ and $(T, \overline{\mathscr{B}(T)}, \bar{m})$ be the completions of $(S, \mathscr{B}(S), P)$ and $(T, \mathscr{B}(T), m)$, respectively. It is easy to see that $\bar{P}$ is tight on $\overline{\mathscr{B}(S)}$.

Let $J: S \rightarrow T$ be continuous and let $\mathscr{U}=\left\{E \subset T: J^{-1}(E)\right.$ is $\bar{P}$-measurable $\}$.

Define a set function $\mu$ on $\mathscr{U}$ by $\mu E=\bar{P}\left(J^{-1}(E)\right)$ where $E \in \mathscr{U}$. $\mathscr{U}$ is a $\sigma$ algebra which contains the Borel sets in $T$ as is easily checked and $(T, \mathscr{U}, \mu)$ is a complete tight measure space. To see that $\mu$ is tight on $\mathscr{U}$, let $E$ be $\mu$-measurable. Then for each positive integer $n$, there exists a compact subset $K_{n}$ of $J^{-1}(E)$ such that $\bar{P}\left(J^{-1}(E) \backslash K_{n}\right)<1 / n$. Put $C_{n}=\bigcup_{i=1}^{n} K_{i}$. Then $\bar{P}\left(J^{-1}(E) \backslash C_{n}\right)<1 / n$. Since $C_{n}$ is compact, $J\left(C_{n}\right)$ is compact and

$$
\mu\left(E \backslash J\left(C_{n}\right)\right)=\bar{P}\left(J^{-1}\left(E \backslash J\left(C_{n}\right)\right)\right) \leq \bar{P}\left(J^{-1}(E) \backslash C_{n}\right)<1 / n .
$$

Hence $\mu$ is tight on $\mathscr{U}$.

LEMMA 2.1. $\mathscr{U}=\overline{\mathscr{B}(T)}$ under the following assumption: $N$ is an $\bar{m}$-null set if and only if $N$ is a $\mu$-null set.

ProOF. It is easy to see that $\overline{\mathscr{B}(T)} \subset \mathscr{U}$. To show that $\mathscr{U} \subseteq \overline{\mathscr{B}(T)}$, let $E \in \mathscr{U}$. Since $\mu$ is tight, there exists a compact set $K_{n}$ such that $K_{n} \subset E$ and $\mu\left(E \backslash K_{n}\right)<1 / n$ for each $n=1,2, \ldots$ Let $K=\bigcup_{n=1}^{\infty} K_{n}$. Then $K$ is a Borel set and $K \subset E$. Since $\mu(E \backslash K) \leq \mu\left(E \backslash K_{n}\right)<1 / n$ for each $n=1,2, \ldots, \mu(E \backslash K)=0$. By assumption, $E \backslash K$ is an $\bar{m}$-null set. Then $E=K \cup(E \backslash K)$ is $\bar{m}$-measurable and so $\mathscr{U} \subset \overline{B(T)}$.

For the measurability theorems in Wiener and Yeh-Wiener spaces in which we are concerned, we have $\overline{\mathscr{B}(T)} \subset \mathscr{U}$. For example, if $E$ is Lebesgue measurable then $J^{-1}(E)$ is Wiener measurable. To establish the converse measurability theorems we need $\mathscr{U}=\overline{\mathscr{B}(T)}$.

THEOREM 2.2. Suppose $\overline{\mathscr{B}(T)} \subset \mathscr{U}$. If there exists an integrable function $g: T \rightarrow R$ such that $g(t)>0$ for $\bar{m}$-a.e. $t$ and $\mu E=\int_{E} g(t) d \bar{m}(t)$ for every $\bar{m}$-measurable set $E$, then $\mathscr{U}=\overline{\mathscr{B}}(T)$.

ProOF. By Lemma 2.1, it suffices to show that if $N$ is a $\mu$-null set then $N$ is an $\bar{m}$-null set. Now we assume that $N$ is a $\mu$-null set and it is not an $\bar{m}$-null set. We consider two cases: (1) $N$ is $\bar{m}$-measurable, (2) $N$ is not $\bar{m}$-measurable. 
If $N$ is $\bar{m}$-measurable, then there exists a Borel set $B \subset N$ such that $m B=$ $\bar{m} N>0$. Since $\mu B=\int_{B} g(t) d \bar{m}(t)>0, \mu_{*} N>0$ where $\mu_{*}$ is the inner measure of $\mu$. Since $\mu N=\mu_{*} N, \mu N>0$ which is a contradiction.

If $N$ is not $\bar{m}$-measurable, i.e., $N \notin \overline{\mathscr{B}(T)}$, then $m B>0$ for every Borel set $B \supset N$. Then $\mu B>0$ for any Borel set $B \supset N$. Since $\mu$ is tight, there exists a compact set $K_{n} \subset N^{c}$ such that $\mu\left(N^{c} \backslash K_{n}\right)<1 / n$ for each $n$. Let $K=\bigcup_{n=1}^{\infty} K_{n}$. Then $K^{c}$ is a Borel set and $N \subset K^{c}$.

$$
\begin{aligned}
\mu\left(K^{c}\right) & =1-\mu(K)=\mu\left(N^{c}\right)-\mu(K) \leq \mu\left(N^{c} \backslash K\right) \\
& \leq \mu\left(N^{c} \backslash K_{n}\right)<1 / n \text { for each } n .
\end{aligned}
$$

Hence $\mu\left(K^{c}\right)=0$. This contradicts the fact that $\mu B>0$ for any Borel set $B \supset N$. Therefore we conclude that every $\mu$-null set is also an $\bar{m}$-null set.

\section{Converse measurability theorems.}

THEOREM 3.1 (KÖEHLER). Let $a=t_{0}<t_{1}<\cdots<t_{n}=b$. Let $E$ be any subset of $\mathbf{R}^{n}$ and let $J$ be defined as in (1). Then $E$ is Lebesgue measurable if and only if $J^{-1}(E)$ is Wiener measurable.

PrOOF. By the definition of Wiener measure, if $E$ is Lebesgue measurable, then $J^{-1}(E)$ is Wiener measurable. To show the converse, let

$$
\mathscr{U}=\left\{E \subset \mathbf{R}^{n} \mid J^{-1}(E) \text { is Wiener measurable }\right\} .
$$

Then $\mathscr{U}$ contains all Lebesgue measurable sets in $\mathbf{R}^{n}$. For $E \in \mathscr{U}$, we define $\mu$ on $\mathscr{U}$ by $\mu E=\nu\left(J^{-1}(E)\right)$. Then every Lebesgue measurable set $E$ satisfies (2). Since Wiener measure is tight on the $\sigma$-algebra of Wiener measurable sets which contains the Borel sets in $C_{1}[a, b],\left(\mathbf{R}^{n}, \mathscr{U}, \mu\right)$ is a complete tight measure space. Hence by Theorem $2.2, J^{-1}(E)$ is Wiener measurable if and only if $E$ is Lebesgue measurable.

Let $a=s_{0}<s_{1}<\cdots<s_{m}=b, \alpha=t_{0}<t_{1}<\cdots<t_{n}=\beta$. Let $E$ be any subset of $\mathbf{R}^{m n}$ and define $J: C_{2}[R] \rightarrow R^{m n}$ by

$$
J(x)=\left(x\left(s_{1}, t_{1}\right), \ldots, x\left(s_{m}, t_{n}\right)\right) .
$$

$J$ is continuous on $C_{2}[R]$ with respect to the uniform topology. By the definition of Yeh-Wiener measure, if $E$ is Lebesgue measurable then $J^{-1}(E)$ is Yeh-Wiener measurable and

$$
\lambda\left(J^{-1}(E)\right)=\int_{E} g(\vec{\xi}) d \gamma(\vec{\xi})
$$

for any Lebesgue measurable set $E$ in $\mathbf{R}^{m n}$, where $\gamma$ is Lebesgue measure on $\mathbf{R}^{m n}$,

$$
\begin{aligned}
& g(\vec{\xi})=\left\{(2 \pi)^{m n}\left\{\left(s_{1}-a\right) \cdots\left(s_{m}-s_{m-1}\right)\left(t_{1}-\alpha\right) \cdots\left(t_{n}-t_{n-1}\right)\right\}^{-1 / 2}\right. \\
& \times \exp \left\{-\frac{1}{2} \sum_{k=1}^{n} \sum_{j=1}^{m}\left(\xi_{j, k}-\xi_{j-1, k}-\xi_{j, k-1}+\xi_{j-1, k-1}\right)^{2} /\left(s_{j}-s_{j-1}\right)\left(t_{k}-t_{k-1}\right)\right\} \\
& >0,
\end{aligned}
$$

and $\vec{\xi}=\left(\xi_{1,1}, \xi_{1,2}, \ldots, \xi_{m, n}\right) \in \mathbf{R}^{m n}$.

We will omit the proof of Theorem 3.2 below since it is similar to the proof given above for Theorem 3.1. 
THEOREM 3.2 (SKOUg). Let $J$ be defined as in (3) and let $E$ be any subset of $\mathbf{R}^{m n}$. Then $E$ is Lebesgue measurable if and only if $J^{-1}(E)$ is Yeh-Wiener measurable.

We shall use the following notation for the Cartesian product of $n$ Wiener spaces $\stackrel{n}{X} C_{1}[a, b]=C_{1}[a, b] \times \cdots \times C_{1}[a, b]$ and $\stackrel{n}{X} \nu=\nu \times \cdots \times \nu$ will denote the product of $n$ Wiener measures on $\stackrel{n}{X} C_{1}[a, b]$.

Let $\alpha=t_{0}<t_{1}<\cdots<t_{n}=\beta$ be a subdivision of $[\alpha, \beta]$. Define $\psi: \stackrel{n}{X} C_{1}[a, b] \rightarrow$ $\stackrel{n}{X} C_{1}[a, b]$ by

$$
\begin{aligned}
& \psi\left(y_{1}, y_{2}, \ldots, y_{n}\right)=\left(\sqrt{\frac{t_{1}-t_{0}}{2}} y_{1}, \sqrt{\frac{t_{1}-t_{0}}{2}} y_{1}+\sqrt{\frac{t_{2}-t_{1}}{2}} y_{2}, \ldots,\right. \\
&\left.\sqrt{\frac{t_{1}-t_{0}}{2}} y_{1}+\cdots+\sqrt{\frac{t_{n}-t_{n-1}}{2}} y_{n}\right) .
\end{aligned}
$$

Then $\psi$ is 1-1 and onto. $\psi$ and $\psi^{-1}$ are continuous with respect to the uniform topology. Let $G: C_{2}[R] \rightarrow \stackrel{n}{X} C_{1}[a, b]$ be defined by $G(x)=\left(x\left(\cdot, t_{1}\right), \ldots, x\left(\cdot, t_{n}\right)\right)$. Then $G$ is a continuous function from $C_{2}[R]$ onto ${ }^{n} X C_{1}[a, b]$. In [1] Cameron and Storvick evaluated certain Yeh-Wiener integrals in terms of Wiener integrals. In particular they obtained the $n$-parallel lines theorem. The converse of the $n$-parallel lines theorem follows quite easily once Theorem 3.3 below is established [2].

THEOREM 3.3. Let $A$ be any subset of $\stackrel{n}{X} C_{1}[a, b]$. Then $\psi^{-1} A$ is $\stackrel{n}{X} \nu$-measurable if and only if $G^{-1} A$ is Yeh-Wiener measurable. Furthermore,

$$
\lambda\left(G^{-1} A\right)=\stackrel{n}{X} \nu\left(\psi^{-1} A\right)
$$

PrOOF. By the $n$-parallel lines theorem, if $\psi^{-1} A$ is $\stackrel{n}{X} \nu$-measurable then $G^{-1} A$ is Yeh-Wiener measurable and $\lambda\left(G^{-1} A\right)=\stackrel{n}{X} \nu\left(\psi^{-1} A\right)$ [2]. To show the converse, let $J=\psi^{-1} \circ G$. Then $J$ is a continuous function from $C_{2}[R]$ onto $\stackrel{n}{X} C_{1}[a, b]$. Let

$$
\mathscr{U}=\left\{E \in \stackrel{n}{X} C_{1}[a, b]: J^{-1}(E) \text { is Yeh-Wiener measurable }\right\}
$$

Define a set function $\mu$ on $\mathscr{U}$ by $\left.\mu E=\lambda\left(J^{-1} E\right)\right)$. For any $\stackrel{n}{X} \nu$-measurable set $E=\psi^{-1}(\psi E), G^{-1}(\psi E)=J^{-1}(E)$ is Yeh-Wiener measurable and $\lambda\left(G^{-1} \psi E\right)=$ $\stackrel{n}{X} \nu\left(\psi^{-1} \psi E\right)=\stackrel{n}{X} \nu(E)$ by the $n$-parallel lines theorem. Hence $E \in \mathscr{U}$ and $\mu E=$ $\lambda\left(J^{-1} E\right)=\lambda\left(G^{-1} \psi E\right)=\stackrel{n}{X} \nu(E)=\int_{E} 1 d \stackrel{n}{X} \nu$. Hence by Theorem 2.2, $\mathscr{U}$ is the $\sigma$ algebra of $\stackrel{n}{X} \nu$-measurable sets. If $G^{-1} A=J^{-1}\left(\psi^{-1} A\right)$ is Yeh-Wiener measurable, then $\psi^{-1} A \in \mathscr{U}$ and so $\psi^{-1} A$ is $\stackrel{n}{X} \nu$-measurable.

ACKNOWLEDGMENT. The authors wish to thank Professors G. W. Johnson and D. L. Skoug for their advice in the writing of this paper. 


\section{REFERENCES}

1. R. H. Cameron and D. A. Storvick, Two related integrals over spaces of continuous functions, Pacific J. Math. 55 (1974), 19-37.

2. K. S. Chang, Converse measurability theorems for Yeh-Wiener space, Pacific J. Math. 97 (1981), $59-63$.

3. K. S. Chang and I. Yoo, A simple proof of converse measurability theorem for Yeh-Wiener spaces, Bull. Korean Math. Soc. 23 (1986), 35-37.

4. K. R. Parthasarathy, Probability measures on metric space, Academic Press, New York, 1967.

5. D. L. Skoug, Converses to measurability theorems for Yeh-Wiener space, Proc. Amer. Math. Soc. 57 (1976), 304-310.

6. J. Yeh, Wiener measure in a space of functions of two variables, Trans. Amer. Math. Soc. 95 (1960), 433-450.

Department of Mathematics, Yonsei University, Seoul, Korea

Department of Mathematics, han Nam University, DaEJon, Korea 